\title{
Prognostic Significance of Hormonal Receptor Status of Malignant Ovarian Tumors
}

\author{
I. G. Tkalia ${ }^{1}$, V. S. Svintsitsky ${ }^{1}$, S. V. Nespryadko ${ }^{1}$, N. Yu. Lukianova ${ }^{2}$, and V. F. Chekhun ${ }^{2}$ \\ 1. National Cancer Institute, Kyiv 03022, Ukraine \\ 2. R. E. Kavetsky Institute of Experimental Pathology, NAS of Ukraine, Kyiv 03022, Ukraine
}

\begin{abstract}
The objective of this study is to investigate hormonal receptor status of MOT (malignant ovarian tumor) and to evaluate its clinical and prognostic significance. Retrospective analysis of the case reports of 284 patients with MOT of different histogenesis, stages I-IV, and immunohistochemical study of paraffin-embedded tissues were performed. Hormonal receptor status of tumors with different morphology genesis was studied and hormonal receptor phenotype of serous OC (ovarian cancer) was determined. The analysis of correlation between the expression of steroid hormone receptors (receptors to estrogens (ER), progesterone (PR) and testosterone (TR)) in ovarian tumors, histological type of tumors and clinical morphological parameters were performed. Overall and relapse-free survival rates of the patients with serous OC depending on the hormonal receptor phenotype of the tumor were assessed. Presence of positive expression of steroid hormone receptors in serous OC (ER-66.4\%, PR-63.4\%, TR-53.0\%), mucinous OC (ER-88.0\%, PR-84.0\%, TR-60.0\%) and in sex cord stromal tumors (ER-74.1\%, PR and TR-77.8\%) is proved by correlation of all steroid receptors expression with morphology type of ovarian tumors $(\mathrm{ER}-\mathrm{r}=0.4$; $\mathrm{PR}-\mathrm{r}=0.4 ; \mathrm{TR}-\mathrm{r}=0.3 ; p<0.05)$. Direct correlation between hormonal receptor phenotype of serous OC and the age period of the patients was established $(r=0.5 ; p=0.002)$ : postmenopausal women patients reported the most increased frequency of serous OC with positive hormonal receptor tumor phenotypes (52.4\%), in particular during their late post-menopausal period (39.0\%). Significantly low overall survival among the patients with positive hormonal receptor phenotype of serous OC was recorded $(29.5 \pm 3.4 \%)$ in comparison with the same score in the patients with negative phenotype of tumors $(44.5 \pm 3.7 \%)(p<0.05)$. Multifactor analysis of Cox-regression model has defined that positive hormonal receptor phenotype of serous OC increases the risk of disease relapse (HR 1.4; 95.0\% CI 1.1-1.7), significantly decreases overall survival rates in the patients (HR 1.4; 95.0\% CI 1.1-1.8). Positive hormonal receptor status of MOT is an independent factor of unfavorable clinical progress of tumor process which can be regarded as the criterion for development of the methods of hormonal therapy application in complex treatment of the patients, and demands further large-scale multi-center studies in that direction.
\end{abstract}

Key words: Malignant ovarian tumors, serous ovarian cancer, hormonal receptor status, estrogen, progesterone, testosterone receptors, phenotype of tumor.

\section{Introduction}

MOT (malignant ovarian tumors) are one of the most complicated problems in oncogynecology $[1,2]$. Complexity of the problem lies in uniqueness of this organ and its role in a woman organism [3, 4]. Multi-component structure of gonad, variety of structures with different functions provides a wide range of histological forms of ovarian tumors and complexity of etiopathogenesis of this disease [5].

Corresponding author: Iuliia G. Tkalia, $\mathrm{PhD}$, research fields: pathogenetic background of hormonal therapy for malignant tumors of ovary.
Hormonal factors play the most important role in pathogenesis of MOT at present time. Ceaseless ovulation owing to pregnancies and childbirths declination in some countries, sterility of different genesis and application of drugs stimulating ovulation belong to them [6-8]. According to the data of literature, ovaries are not only producing sex steroid hormones, but also act as a target tissue for them, the same as the other hormone-dependent organs [9, 10]. Estrogens, progesterone and testosterone, the same as receptors to them, are promoters of all hormone-dependent tumors, in particular, of breast 
cancer (BC), endometrial and ovarian (OC) cancer $[11,12]$.

Therapy strategy for patients with MOT includes surgical component and chemotherapy. Despite improvement of surgical treatment strategies and application of modern chemotherapy schemes [2-15], long-term results of treatment in patients with disseminated MOT remain unsatisfactory $[16,17]$.

For many years, hormonal therapy for patients with progressing and chemo-resistant MOT has been empirically prescribed as a "despair therapy" demonstrating the low rate of efficacy [18, 19]. Clinical interest in hormonal treatment of OC patients was increased due to development of molecular-biological technologies and possibility to determine receptors to estrogens (ER), progesterone (PR) and androgens in tumor tissue [20]. However, criteria of hormone-dependency of MOT and application of hormonal therapy in combined treatment of patients, the same as prognosis depending on expression of steroid receptors are remaining disputable.

Prognostic value of HRS (hormonal receptor status) of MOT has been studied for many years. Certain studies have determined high survival of OC patients at expression of ER and PR in tumor [21-23]. Other studies showed that expression of PR and receptor to testosterone (TR) are favorable prognostic factors, and expression of ER is associated with progression of disease and short relapse-free period [24-31].

Interrelation between HRS and degree of tumor differentiation as well as impact of chemotherapy on $\mathrm{ER}, \mathrm{PR}, \mathrm{TR}$ in tumor tissue of ovary has not been determined yet [10, 32].

Thus, the question concerning the mechanisms for realization of systemic-local dyshormonal disorders causing MOT and clinical significance of ovarian tumors receptor status till this day remains open [33]. Solution of this question will allow us to specify MOT pathogenesis, prognosis of disease, but also to substantiate indications for hormone therapy carrying out as a component of combined treatment for the patients.

The aim of the research was to study hormonal receptor status of MOT of different genesis and to determine its clinical significance in patients with serous OC.

\section{Material and Methods}

The results of retrospective analysis of case histories in 284 patients with MOT of different genesis (stage I-IV) and paraffin blocks of postsurgical material have been taken as material for this study. Patients were treated in National Cancer Institute within 2001-2009. All patients underwent treatment by radical program: surgical, combined and complex (according to the standards of diagnostics and treatment for cancer patients approved by the orders at $\mathrm{MH}$ of Ukraine № 226 from 27.07.1998 and № 554 from 17.09.2007). Combined treatment consisted in cytoreductive surgery followed by adjuvant chemotherapy or in combination with neoadjuvant chemotherapy. Complex treatment included surgical intervention, chemotherapy and radiation. Informed consent was received from all of the patients participating in the study.

MOT diagnosis has been verified using morphological study of post-surgical material according to the histological classification of ovarian tumors (2002), clinical staging of MOT-by international FIGO classification (2009) [34].

Immunohistochemical study of ER, PR, and TR in ovarian tumor cells has been carried out on paraffin sections 4-5 micron thickness, which were placed on glasses processed with poly-L-lysine. As primary antibodies, monoclonal antibodies specific to ER, PR and TR have been used (anti-Human Estrogen Receptor alfa Clone 1D5 (DakoCytomation, Denmark), anti-Human Progesterone Receptor Clone PgR 636 (DakoCytomation, Denmark), testosterone antibody Clone GTX72779 (GeneTex, USA)). For 
visualization of the results of reaction, kit of reagents En Vision system (DakoLSAB2system, Denmark) has been used in accordance with recommendations of the manufacturer; sections were stained by Meyer's hematoxylin. For evaluation of immunohistochemical expression of ER, PR and TR, semi-quantitative method has been applied. At presence of specific nuclear staining, the quantity of immune-positive and immune-negative cells in percentage has been determined. In each histological specimen, expression of steroid receptors in 1000 tumor cells has been analyzed. Since till this day no conventional semi-quantitative methods of total assessment of HRS for MOT exist, method which is mostly found in data of literature [35], has been taken as a model. Degree of steroid receptor expression has been taken in scores: 0 score —no staining of nuclei of tumor cells; 1 score-poor staining $\leq 10 \%$; 2 scores-moderate staining-11-50\%; 3 scores—high staining-51-80\%; 4 scores-hyperexpression $\longrightarrow 81 \%$ of cells. The quantity of stained nuclei with more than $10 \%$ of moderate and high level of staining has been regarded as a positive expression of steroid receptors. As positive control, monoclonal antibodies against pan-cytokeratins have been used; as negative control-buffered physiological solution has been applied to histological sections instead of monoclonal antibodies.

Results of studies have been evaluated depending on women age periods: reproductive (up to 45), perimenopause (46-55), early postmenopause (56-60) and late postmenopause (61 and more) according to the WHO classification (1980) [36]. Overall and relapse-free survival of patients with serous OC depending on tumor HRS has been determined. Correlation between HRS and stage, tumor differentiation grade has been assessed. Influence of neoadjuvant chemotherapy on expression of steroid hormone receptors in serous OC has been analyzed.

Statistical analysis of obtained data consisted in use of nonparametric statistics. For comparison of studied groups, $U$-criterion of Whitney-Mann has been applied. Correlation has been evaluated using Gamma rank correlation. Survival of patients has been analyzed by Kaplan-Meier method and log-rank criterion. Analysis of prognostic factors was performed by use of Cox-regression model. Statistically significant were considered data at $p<$ 0.05 . Processing of the results of study has been carried out using program package STATISTICA 6.0.

\section{Results}

3.1 Characteristics of the Patients with MOT of Different Histogenesis according to Clinical-Morphological and Immunohistochemical Indices of Tumors

Analysis of case histories has demonstrated that age of the patients with MOT $(\mathrm{n}=284)$ of different genesis varied from 16 to 82 , mean age $52.8 \pm 2.2$. By histological structure of tumors, patients were allocated (Table 1) into serous OC (232/81.7\%), mucinous OC (25/8.8\%) and sex cord-stromal tumors (SCST) (27/9.5\%). Mean age of patients was equal to (51.7, 52.8 and 53.9, respectively).

Patients with MOT, who were prescribed neoadjuvant chemotherapy, depending on genesis of tumors, have been divided as follows: serous OC has been diagnosed in $84(36.2 \%)$ patients, mucinous OC-in $2(7.4 \%)$ and SCST - in 4 (16.0\%). Grade of differentiation of ovarian tumors is represented in Table 1. Patients with serous OC and SCST had high percentage of tumors with low differentiation (49.1 and $59.3 \%$, respectively). In patients with mucinous OC most tumors were moderately differentiated $(40.0 \%)$.

Analysis of MOT spreading has shown that a number of patients with stage III was the highest $-73.6 \%$ ( $\mathrm{n}=$ 209). Data on distribution of patients by stages depending on histological MOT type are represented in Table 2. As seen from Table 2, expression of steroid hormones in MOT was variable. Positive expression of ER was observed in $66.4 \%$ of patients 
Table 1 Distribution of patients by age, MOT histology, grade of tumor differentiation $(\mathbf{n}=\mathbf{2 8 4})$.

\begin{tabular}{|c|c|c|c|c|c|c|c|c|c|}
\hline \multirow{3}{*}{$\begin{array}{l}\text { Histological type } \\
\text { of tumor }\end{array}$} & \multirow{2}{*}{\multicolumn{2}{|c|}{ Number of patients }} & \multirow{3}{*}{ Mean age/range, years } & \multicolumn{6}{|c|}{ Tumor differentiation grade } \\
\hline & & & & \multicolumn{2}{|c|}{ High } & \multicolumn{2}{|c|}{ Moderate } & \multicolumn{2}{|c|}{ Low } \\
\hline & $\mathrm{n}$ & $\%$ & & $\mathrm{n}$ & $\%$ & $\mathrm{n}$ & $\%$ & $\mathrm{n}$ & $\%$ \\
\hline Serous OC & 232 & 100.0 & $51.7 \pm 0.8 / 18-82$ & 45 & 19.4 & 73 & 31.5 & 114 & 49.1 \\
\hline Mucinous OC & 25 & 100.0 & $52.8 \pm 3.2 / 16-69$ & 7 & 28.0 & 10 & 40.0 & 8 & 32.0 \\
\hline SCST & 27 & 100.0 & $53.9 \pm 2.5 / 27-70$ & 3 & 11.1 & 8 & 29.6 & 16 & 59.3 \\
\hline
\end{tabular}

Table 2 Frequency of receptor-positive MOT of different genesis depending on stage of disease (by ER, PR and TR), (n = 284).

\begin{tabular}{lllllll}
\hline Number of patients with receptor-positive tumor & \multicolumn{3}{c}{ ER +} & & & PR + \\
\hline MOT histology/stages & $\mathrm{n}$ & $\%$ & $\mathrm{n}$ & $\%$ & $\mathrm{n}$ & $\%$ \\
\hline Serous OC, & 154 & 66.4 & 147 & 63.4 & 123 & 53.0 \\
$\mathrm{~N}=232(100.0 \%)$ & 20 & 87.0 & 17 & 73.9 & 13 & 56.5 \\
Stage I, $\mathrm{n}=23(100.0 \%)$ & 11 & 84.6 & 11 & 84.6 & 2 & 15.4 \\
Stage II, $\mathrm{n}=13(100.0 \%)$ & 116 & 62.7 & 113 & 61.1 & 103 & 55.7 \\
Stage III, $\mathrm{n}=185(100.0 \%)$ & 7 & 63.6 & 6 & 54.5 & 5 & 45.5 \\
Stage IV, $\mathrm{n}=11(100.0 \%)$ & 22 & 88.0 & 21 & 84.0 & 15 & 60.0 \\
Mucinous OC, & 9 & 90.0 & 8 & 80.0 & 6 & 60.0 \\
$\mathrm{n}=25(100.0 \%)$ & 13 & 86.7 & 13 & 86.7 & 9 & 60.0 \\
Stage I-II, $\mathrm{n}=10(100.0 \%)$ & 20 & 74.1 & 21 & 77.8 & 21 & 77.8 \\
Stage III, $\mathrm{n}=15(100.0 \%)$ & 11 & 64.7 & 11 & 64.7 & 15 & 88.2 \\
Sex cord-stromal tumors (SCST), & 9 & 90.0 & 10 & 100.0 & 6 & 60.0 \\
$\mathrm{n}=27(100.0 \%)$ & & & &
\end{tabular}

with serous OC, PR - in 63.4\%, TR - in 53.0\% (Figs. 1-3 and Table 2). Positive expression of the same markers has been detected in patients with mucinous OC- $-88.0 \%, 84.0 \%$ and $60.0 \%$, respectively. Positive staining of cells of SCST has been observed in $74.1 \%$ of patients for ER and $77.8 \%$ - for RP and TR. It should be mentioned that the highest percentage of receptor-positive tumors has been determined in patients with mucinous OC and SCST. Statistical analysis has demonstrated significant rank correlation between morphological structure of tumors and expression of steroid hormone receptors: for ER $-\mathrm{r}=$ $0.364(p=0.002), \mathrm{RP}-\mathrm{r}=0.408(p<0.001)$ and TR $-\mathrm{r}=0.289(p=0.0048)$ (Table 5). When comparing frequencies of expression of steroid hormone receptors in serous OC and SCST, reliable differences have been established only for TR $(p=0.016)$. When comparing frequency of expression of steroid hormone receptors in serous and mucinous OC, differences for ER and PR have been marked ( $p=$ 0.027 and $p=0.039$, respectively), but when comparing frequencies of expression of ER, PR and TR in mucinous OC and SCST, no significant differences were found.

Weak correlation between frequency of expression of ER, PR in tumors of different genesis and stage of disease has been established: $r=-0.304(p=0.002)$ and $r=-0.207(p=0.027)$, respectively (Tables 2 and $3)$. In patients with serous $\mathrm{OC}$, at increase of disease stage, tendency to a decline of expression frequency for ER and PR has been determined, while in patients with mucinous OC and SCST tendency to elevation of expression frequency for these receptors has been observed. No connection between TR expression in tumors of different genesis and stage of tumor process has been noticed $(r=-0.053, p=0.538)$.

Statistical analysis has not detected significant correlation between frequency of receptor-positive MOT 


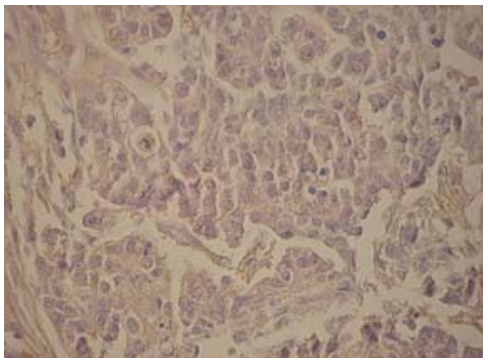

(a)

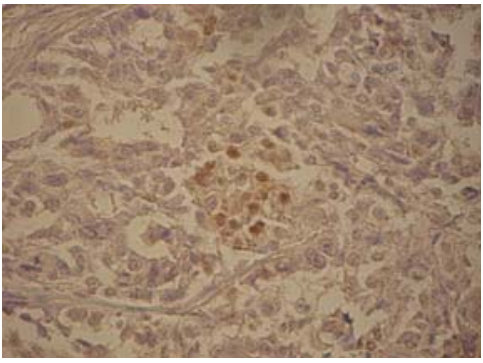

(b)

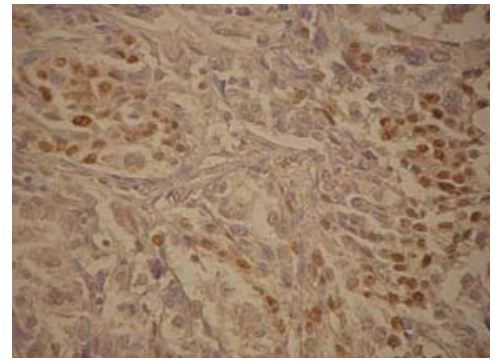

(c)

Fig. 1 Serous OC. Immunohistochemical expression of ER in tumor cells, $\times 400$. $\mathrm{a}$-no expression; $\mathrm{b}$ - low expression of ER; $\mathrm{c}$ — - high expression of ER.

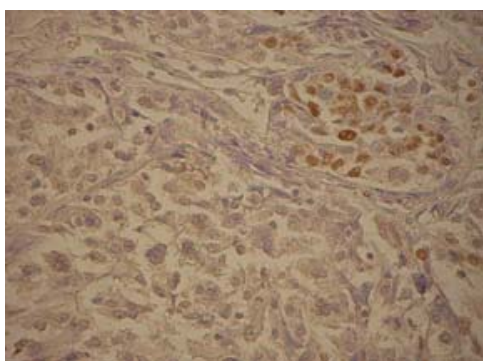

(a)

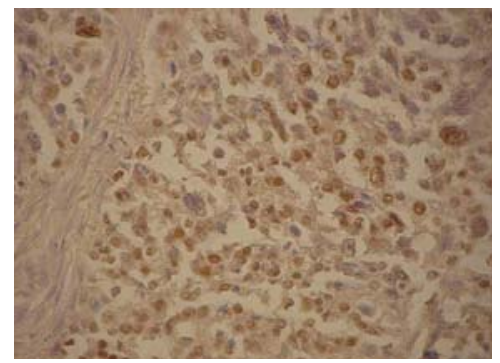

(b)

Fig. 2 Serous OC. Immunohistochemical expression of PR in tumor cells, $\times 400$. a-low expression of PR; b-high expression of PR.

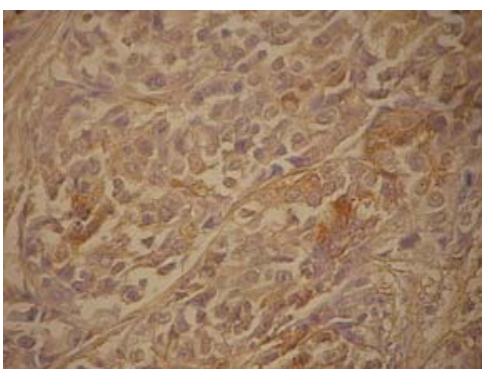

(a)

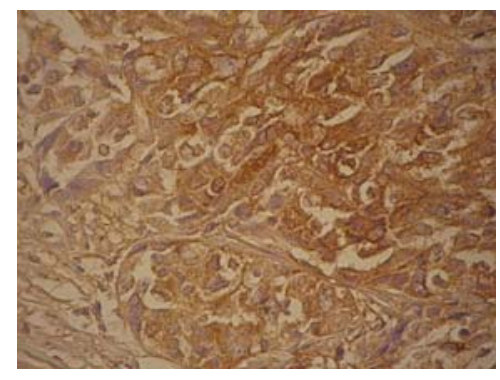

(b)

Fig. 3 Serous OC. Immunohistochemical expression of TR in tumor cells, $\times 400$. a-low expression of TR; b-high expression of TR.

Table 3 Gamma rank correlations between expression of steroid hormone receptors, histological type and tumor differentiation grade, stage of disease in patients with MOT, $(\mathbf{n}=\mathbf{2 8 4})$.

\begin{tabular}{lllllll}
\hline Index & Stage & Histological type of tumor & Differentiation of tumor & ER & PR & TR \\
\hline Stage & 1.000 & $\mathbf{- 0 . 5 8 7} *$ & 0.128 & $\mathbf{- 0 . 3 0 4 *}$ & $\mathbf{- 0 . 2 0 7 *}$ & -0.053 \\
Histological type of tumor & $\mathbf{- 0 . 5 8 7 *}$ & 1.000 & -0.069 & $\mathbf{0 . 3 6 4 *}$ & $\mathbf{0 . 4 0 8 *}$ & $\mathbf{0 . 2 8 9 *}$ \\
Differentiation of tumor & 0.128 & -0.069 & 1.000 & -0.066 & 0.020 & 0.054 \\
ER & $\mathbf{- 0 . 3 0 4} *$ & $\mathbf{0 . 3 6 4 *}$ & -0.066 & 1.000 & $\mathbf{0 . 9 2 8 *}$ & $\mathbf{0 . 3 9 8}$ \\
PR & $\mathbf{- 0 . 2 0 7 *}$ & $\mathbf{0 . 4 0 8 *}$ & 0.020 & $\mathbf{0 . 9 2 8 *}$ & 1.000 & $\mathbf{0 . 3 5 4}$ \\
TR & -0.053 & $\mathbf{0 . 2 8 9 *}$ & 0.054 & $\mathbf{0 . 3 9 8 *}$ & $\mathbf{0 . 3 5 4 *}$ & 1.000 \\
\hline
\end{tabular}

* Correlation coefficient significant at the level of $p<0.05$.

of different genesis (Table 3) and grade of MOT differentiation: for $\mathrm{ER}-p=0.382, \mathrm{PR}-p=0.789$ and TR $-p=0.444$. However, significant correlation of influence of chemotherapy on expression of PR ( $p$ $=0.001)$ and lack of influence on expression of ER and TR ( $p=0.091$ and $p=0.787$, respectively) have 
been determined. When analyzing dependence of expression of steroid receptors between each other, rank correlation between ER and PR $(p<0.0001)$, ER and TR $(p<0.001)$, PR and TR $(p<0.001)$ was reported that was especially significant between ER and PR with $r=0.928$.

Thus, positive HRS was found not only in serous, but also in mucinous OC and SCST, the highest percentage of receptor-positive tumors at that was determined in patients with mucinous $\mathrm{OC}$, which is characterized by unfavorable clinical course of tumor process, and in patients with SCST, which are known to have high hormonal activity [2, 37]. It was confirmed by the determined correlation between morphological structure of tumors and expression of steroid hormone receptors. Represented results have shown that ovary with malignant tumor of different histogenesis is a target tissue for systemic-local classic and non-classic steroids irrespective of grade of differentiation of tumor tissue.

\subsection{Study of Hormonal-Receptor Status of Serous OC} and its Correlation with Clinical-Morphological Indices of Tumors

The most numerous group in our study consisted of patients with serous OC $(n=232)$, therefore, the further analysis has been carried out for this category of patients. Mean period of observation of patients with serous OC was $39.5 \pm 1.7$ months, mean relapse-free period-33.7 \pm 1.8 months, median of relapse-free survival $-30.0 \pm 2.1$ months. Out of total number of patients, $28.4 \%$ were in reproductive period, $31.0 \%$-in perimenopausal and $40.5 \%$-in postmenopausal period (in early postmenopausal-15.1\%, in late menopausal-25.4\%). In $149(64.2 \%)$ patients, the relapse of disease has been determined. Patients with relapse of disease depending on age period have been divided as follows: $30(20.1 \%)$ patients of reproductive period, 55 (36.9\%)-perimenopause, number of patients of postmenopausal period was the highest- $64(42.0 \%)$, among them-22 (14.8\%) patients in early menopause, and $42(28.2 \%)$ - late postmenopause. It should be mentioned that the highest number of relapses in patients with $\mathrm{OC}$ has been observed in perimenopause and in postmenopause that indicates the most aggressive course of disease and unfavorable prognosis.

When analyzing expression of steroid hormone receptors in serous OC depending on age of patients (Table 4), we have noticed that mostly estrogen-, progesterone-, testosterone-receptor-positive tumors have been observed in late postmenopause- $79.7 \%$, 79.7 and $59.3 \%$, respectively. Significant rank correlation (Table 5) between frequency of expression of ER, PR and age of patients has been established ( $r=-0.253$ at $p<0.001$ and $r=-0.173$ at $p=0.021$, respectively). Correlation coefficient for TR composed -0.076 at $p=0.297$ (Table 5), that is an evidence of absence of this factor correlation with age of patients. The second place by frequency of receptor-positive tumors belongs to the patients of perimenopausal age. As it has been determined earlier,

Table 4 The frequency of expression of ER, PR and TR in patients with serous OC depending on age period (n= 232).

\begin{tabular}{|c|c|c|c|c|c|c|c|c|}
\hline \multirow{3}{*}{ Age period } & \multicolumn{8}{|c|}{ Number of patients with receptor-positive tumor } \\
\hline & \multicolumn{2}{|c|}{$\mathrm{ER}+$} & \multicolumn{2}{|c|}{$\mathrm{PR}+$} & \multicolumn{2}{|c|}{$\mathrm{TR}+$} & \multicolumn{2}{|c|}{ Total } \\
\hline & $\mathrm{n}$ & $\%$ & $\mathrm{n}$ & $\%$ & $\mathrm{n}$ & $\%$ & $\mathrm{n}$ & $\%$ \\
\hline Reproductive period & 42 & 63.6 & 35 & 53.0 & 32 & 48.5 & 66 & 100.0 \\
\hline Perimenopause & 46 & 63.9 & 43 & 59.7 & 41 & 56.9 & 72 & 100.0 \\
\hline Postmenopause & 66 & 70.2 & 69 & 73.4 & 51 & 54.3 & 94 & 100.0 \\
\hline Early postmenopause & 19 & 54.3 & 22 & 62.9 & 16 & 45.7 & 35 & 100.0 \\
\hline Late postmenopause & 47 & 79.7 & 47 & 79.7 & 35 & 59.3 & 59 & 100.0 \\
\hline Total & 154 & & 147 & & 124 & & 232 & \\
\hline
\end{tabular}


Table 5 Gamma rank correlations between steroid hormone receptors, phenotype of tumor, its grade of differentiation, stage of disease, neoadjuvant chemotherapy, age period of patients with serous OC $(n=232)$.

\begin{tabular}{lcc}
\hline Rate & Correlation coefficient & $p$ \\
\hline ER \& stage & $\mathbf{- 0 . 4 2 0}$ & $\mathbf{0 . 0 0 0 5}$ \\
ER \& tumor differentiation grade & -0.118 & 0.1470 \\
ER \& neoadjuvant chemotherapy & -0.154 & 0.1056 \\
ER \& PR & $\mathbf{0 . 9 1 3}$ & $\mathbf{0 . 0 0 0 1}$ \\
ER \& TR & $\mathbf{0 . 3 6 2}$ & $\mathbf{0 . 0 0 0 1}$ \\
ER \& age period & $\mathbf{- 0 . 2 5 3}$ & $\mathbf{0 . 0 0 0 9}$ \\
\hline PR \& stage & $\mathbf{- 0 . 3 1 0}$ & $\mathbf{0 . 0 0 6 2}$ \\
PR \& tumor differentiation grade & -0.060 & 0.4565 \\
PR \& neoadjuvant chemotherapy & $\mathbf{- 0 . 3 1 6}$ & $\mathbf{0 . 0 0 0 5}$ \\
PR \& ER & $\mathbf{0 . 9 1 3}$ & $\mathbf{0 . 0 0 0 1}$ \\
PR \& TR & $\mathbf{0 . 3 7 4}$ & $\mathbf{0 . 0 0 0 1}$ \\
PR \& age period & $\mathbf{0 . 1 7 3}$ & $\mathbf{0 . 0 2 0 5}$ \\
\hline TR \& stage & 0.176 & 0.0902 \\
TR \& tumor differentiation grade & 0.045 & 0.5632 \\
TR \& neoadjuvant chemotherapy & 0.041 & 0.6528 \\
TR \& ER & $\mathbf{0 . 3 6 2}$ & $\mathbf{0 . 0 0 0 1}$ \\
TR \& PR & $\mathbf{0 . 3 7 4}$ & $\mathbf{0 . 0 0 0 1}$ \\
TR \& age period & -0.076 & 0.2968 \\
\hline tumor phenotype \& stage & 0.118 & 0.1531 \\
tumor phenotype \& tumor differentiation grade & 0.031 & 0.6120 \\
tumor phenotype \& neoadjuvant chemotherapy & 0.117 & 0.1063 \\
tumor phenotype \& age period & $\mathbf{0 . 1 8 0}$ & $\mathbf{0 . 0 0 1 7}$ \\
\hline
\end{tabular}

patients with serous OC in the exactly perimenopausal and postmenopausal periods have most aggressive course and unfavorable prognosis of the disease $[4,5$, $10,11]$. In postmenopausal period taken as a whole, ER at serous OC has been detected in $70.2 \%$ of patients, PR-in $73.4 \%$, and $\mathrm{TR}-\mathrm{in} 54.3 \%$. The lowest percentage of receptor-positive tumors has been observed in patients of reproductive age.

As seen in Table 5, there is no rank correlation between frequency of expression of steroid hormone receptors and grade of differentiation of tumors. However, it has been determined that neoadjuvant chemotherapy influences only $\mathrm{PR}$ expression $(p=$ 0.0005), but, at the same time, has no influence on the concentration of ER and TR $(p=0.1056$ and $p=$ 0.6528 , respectively). In serous OC, the same as in all MOT, we have registered significant correlation between expression of ER and PR $(r=0.913$ at $p<$ $0.0001)$, ER and TR $(r=0.362$ at $p<0.0001)$, PR and TR $(r=0.374$ at $p<0.0001)$.

\subsection{Analysis of Serous OC According to Hormonal Receptor Phenotype}

As seen in Table 6, the highest number of patients with serous OC had tumor phenotype, which was positive by all steroid hormone receptors $(82 / 35.3 \%)$. The second place by frequency was taken by the group of patients with tumor phenotype, which was positive only by ER and PR (49/21.1\%). The third and fourth places belong to the patients with negative tumor phenotype by all receptors (37/15.9\%) and phenotype which was positive only by TR $(25 / 10.8 \%)$. The fact was of interest that the highest number of patients with tumor phenotype $\mathrm{ER}+\mathrm{PR}+\mathrm{TR}+$ has been observed in late postmenopause- $39.0 \%$, and in postmenopausal period taken as a whole, the number of patients with such tumor phenotype constituted 43 $(52.4 \%)$. The lowest percentage of patients with mentioned phenotype has been registered in reproductive age $-20.7 \%$. Tumor phenotype ER+PR+TR- 
Table 6 Distribution of patients with serous OC by tumor phenotype and age period $(n=232)$.

\begin{tabular}{|c|c|c|c|c|c|c|c|c|c|c|}
\hline \multirow{3}{*}{ Tumor phenotype } & \multirow{2}{*}{\multicolumn{2}{|c|}{ Number of patients }} & \multicolumn{8}{|c|}{ Age periods } \\
\hline & & & \multicolumn{2}{|c|}{ Reproductive } & \multicolumn{2}{|c|}{ Perimenopause } & \multicolumn{2}{|c|}{$\begin{array}{c}\text { Early } \\
\text { postmenopause }\end{array}$} & \multicolumn{2}{|c|}{$\begin{array}{c}\text { Late } \\
\text { postmenopause }\end{array}$} \\
\hline & n (\%) & $\%$ & $\mathbf{n}$ & $\%$ & $\mathbf{n}$ & $\%$ & $\mathbf{n}$ & $\%$ & $\mathbf{n}$ & $\%$ \\
\hline ER+PR+TR+ & $82(35.3)$ & 100.0 & 17 & 20.7 & 22 & 26.9 & 11 & 13.4 & 32 & 39.0 \\
\hline ER+PR+TR- & 49 (21.1) & 100.0 & 17 & 34.7 & 15 & 30.6 & 6 & 12.2 & 11 & 22.5 \\
\hline ER+PR-TR+ & $10(4.3)$ & 100.0 & 5 & 50.0 & 4 & 40.0 & 0 & 0.0 & 1 & 10.0 \\
\hline ER+PR-TR- & $13(5.6)$ & 100.0 & 3 & 23.1 & 5 & 38.5 & 2 & 15.4 & 3 & 23.1 \\
\hline ER-PR+TR+ & $7(3.0)$ & 100.0 & 0 & 0.0 & 5 & 71.4 & 2 & 28.6 & 0 & 0.0 \\
\hline ER-PR+TR- & $9(3.9)$ & 100.0 & 1 & 11.1 & 1 & 11.1 & 3 & 33.3 & 4 & 44.4 \\
\hline ER-PR-TR+ & $25(10.8)$ & 100.0 & 10 & 40.0 & 10 & 40.0 & 3 & 12.0 & 2 & 8.0 \\
\hline ER-PR-TR- & 37 (15.9) & 100.0 & 13 & 35.1 & 10 & 27.0 & 8 & 21.6 & 6 & 16.2 \\
\hline Total & \multicolumn{2}{|l|}{$232(100.0)$} & \multicolumn{2}{|c|}{$n=66$} & \multicolumn{2}{|c|}{$\mathrm{n}=72$} & $\mathbf{n}=$ & & \multicolumn{2}{|c|}{$\mathrm{n}=59$} \\
\hline
\end{tabular}

was in an equal number of patients in postmenopausal and in reproductive period by 17 (34.7\%) and only in perimenopause such a tumor phenotype has been detected in $15(30.6 \%)$ patients. It should be mentioned that the highest number of reproductive age patients had tumor phenotype ER-PR-TR- (13/35.1\%), and in late postmenopause this phenotype has been observed only in $16.2 \%$ of patients. When comparing patients of reproductive and late postmenopausal periods, significant differences for the phenotype of serous OC ( $p=0.004)$ have been determined, such significant differences were established between patients in perimenopause and late postmenopause ( $p=$ 0.009). When comparing tumor phenotype of patients of reproductive, perimenopausal and early postmenopausal periods, no significant differences were found ( $p=0.850$ and $p=0.873$, respectively). Also, there are no significant differences by tumor phenotype between patients of perimenopause and early postmenopause $(p=0.739)$.

\subsection{Evaluation of Survival in Patients with Serous OC} Depending on Hormonal Receptor Status and Its Prognostic Significance

We have carried out univariate analysis of overall and relapse-free survival of patients with serous OC depending on expression of each steroid hormone receptor (Figs. 4-9). Rates of overall and relapse-free survival of patients are represented in Table 7. Data in
Table 7 demonstrate that 3-year survival rate in patients with serous receptor-negative tumor to estrogens and progesterone was higher than in patients with receptor-positive tumor, but differences between them were insignificant $(p=0.594$ and $p=0.452$, respectively), since 5-year survival rate in two groups of patients turned out to be the same and equaled $39.3 \%$. Results of 3-year and 5-year survival rates in patients with receptor-negative tumor to testosterone (Fig. 6, Table 7) were interesting. They turned out to be significantly higher than in patients with receptor-positive tumors $(p=0.0345)$. It should be mentioned that median of relapse-free survival in patients with receptor-negative tumor concerning all steroid hormones was higher than in patients with receptor-positive tumors, but significant rate has been determined only for TR $(p<0.0500)$.

Hereafter, differences in 3-year and 5-year overall survival of patients with serous OC depending on tumor phenotype have been analyzed (Fig. 10). Statistically significant intergroup differences in survival of patients with the following phenotypes have been determined: $\mathrm{ER}+\mathrm{PR}+\mathrm{TR}+$ and ER-PR-TR- $(p=0.006)$, between $E R+P R+T R+$ and $\mathrm{ER}+\mathrm{PR}+\mathrm{TR}-(\mathrm{p}=0.0150)$, between $\mathrm{ER}+\mathrm{PR}+\mathrm{TR}+$ and ER-PR-TR+ $(p<0.050)$. For this reason, we have evaluated survival of patients who had above-listed phenotypes of serous OC only. Patients with tumor phenotype which was negative by all steroid hormone 


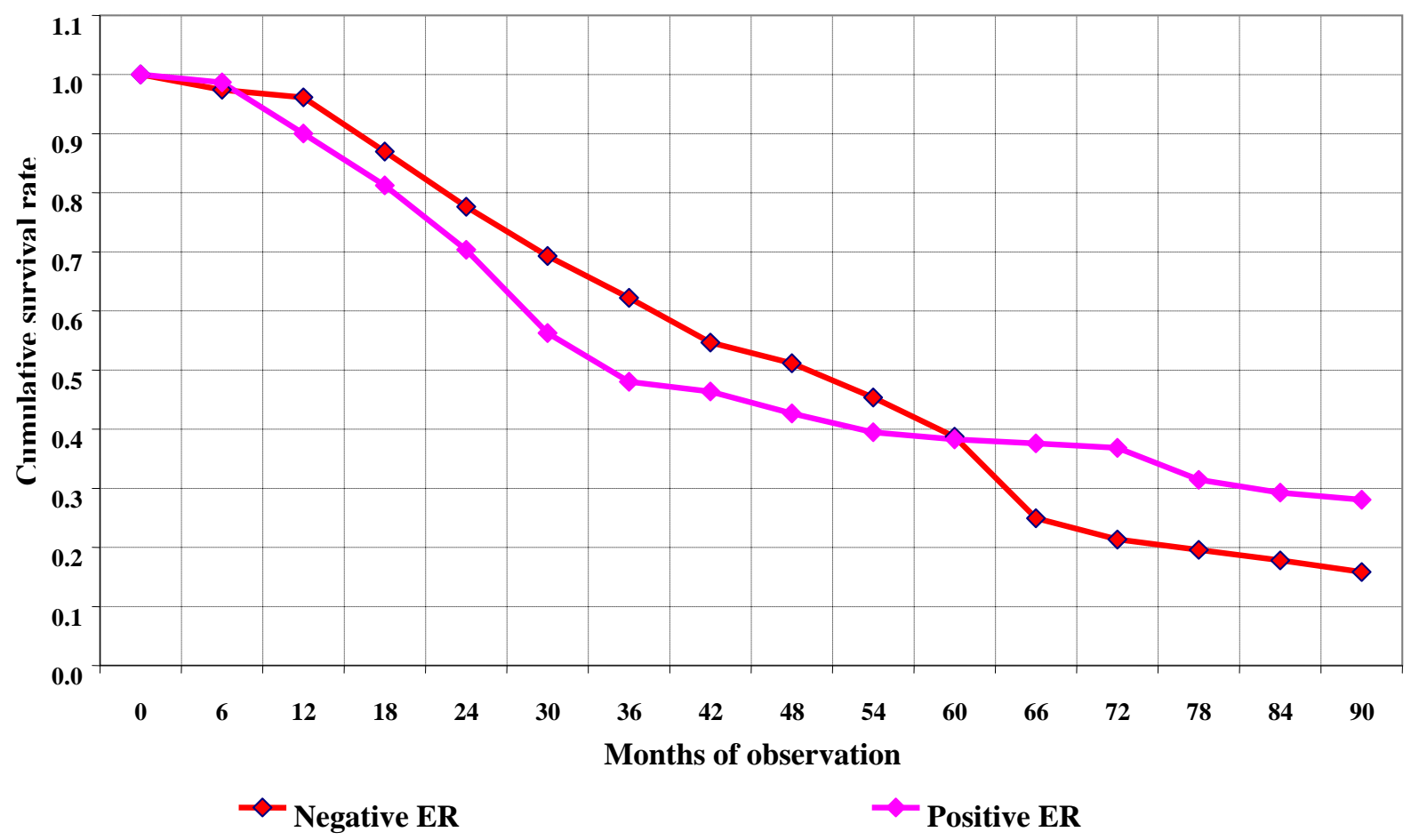

Fig. 4 Overall survival of patients with serous OC with expression of estrogen receptors Kaplan-Meier survival curves, log-rank criterion, $p=0.594$.

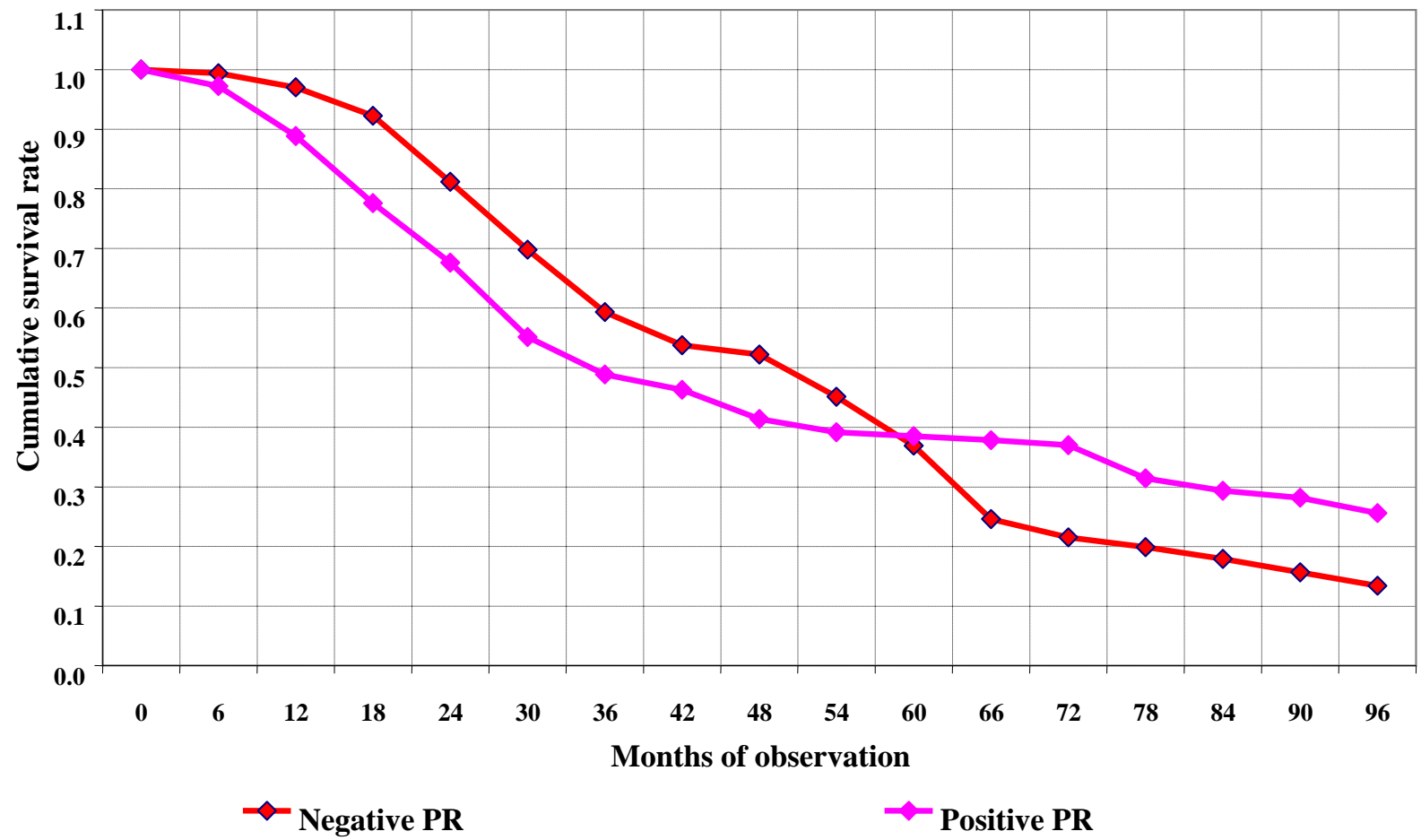

Fig. 5 Overall survival of patients with serous OC with expression of progesterone receptor. Kaplan-Meier survival curves, log-rank criterion, $p=0.452$. 

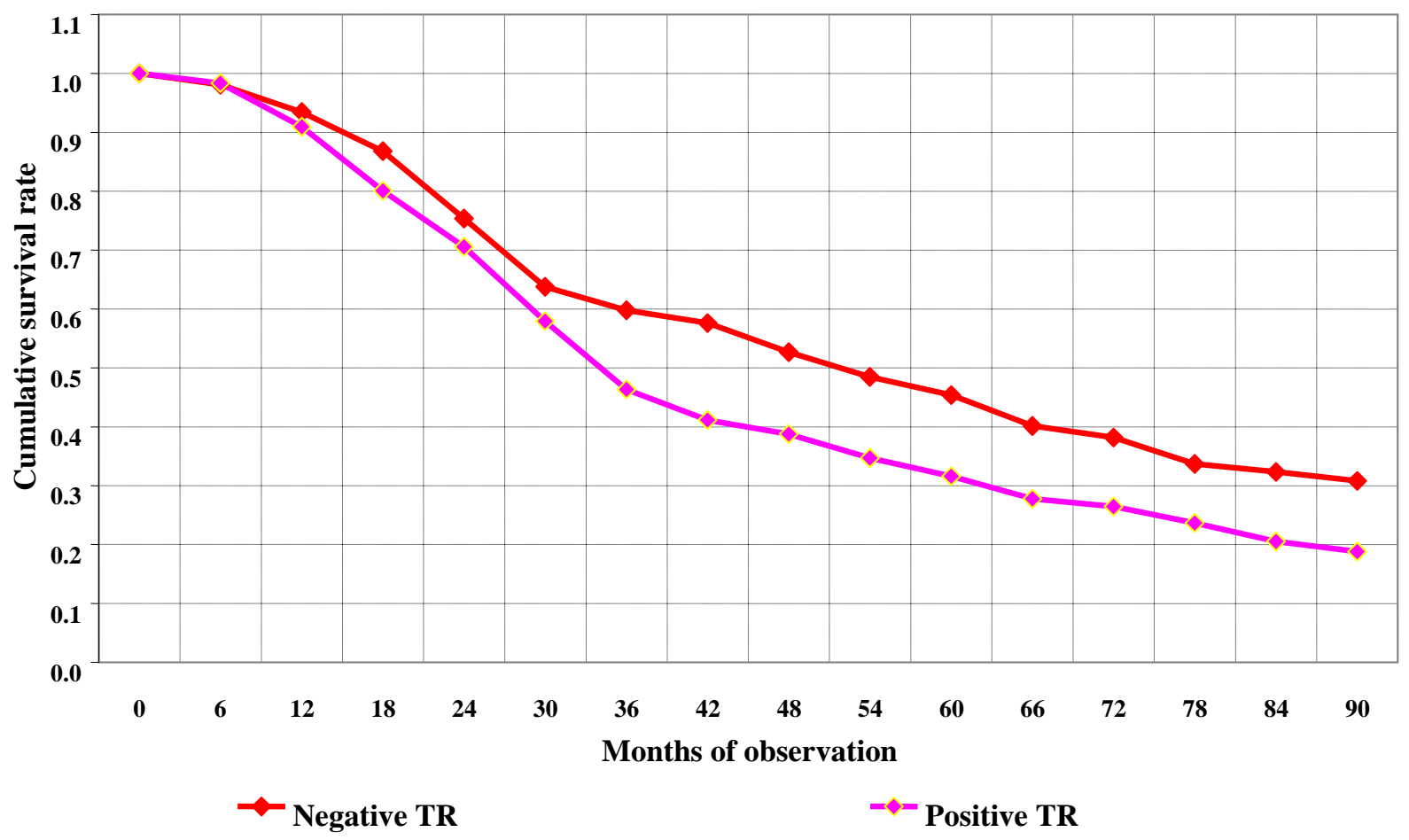

Fig. 6 Overall survival of patients with serous OC with expression of testosterone receptor. Kaplan-Meier survival curves, log-rank criterion, $p=0.0345$.

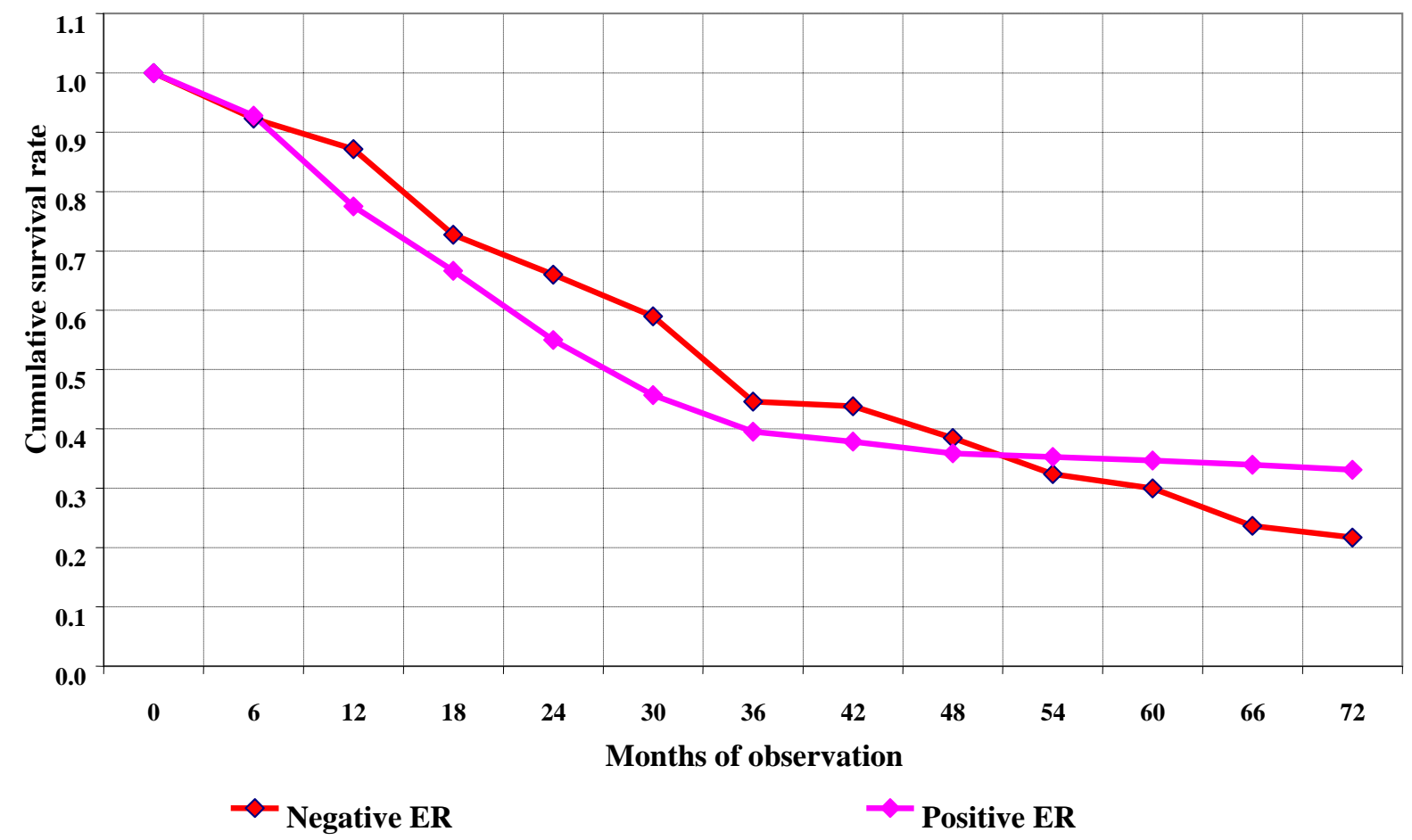

Fig. 7 Relapse-free survival of patients with serous OC with expression of estrogens receptor. Kaplan-Meier survival curves, log-rank criterion, $p=0.6876$. 


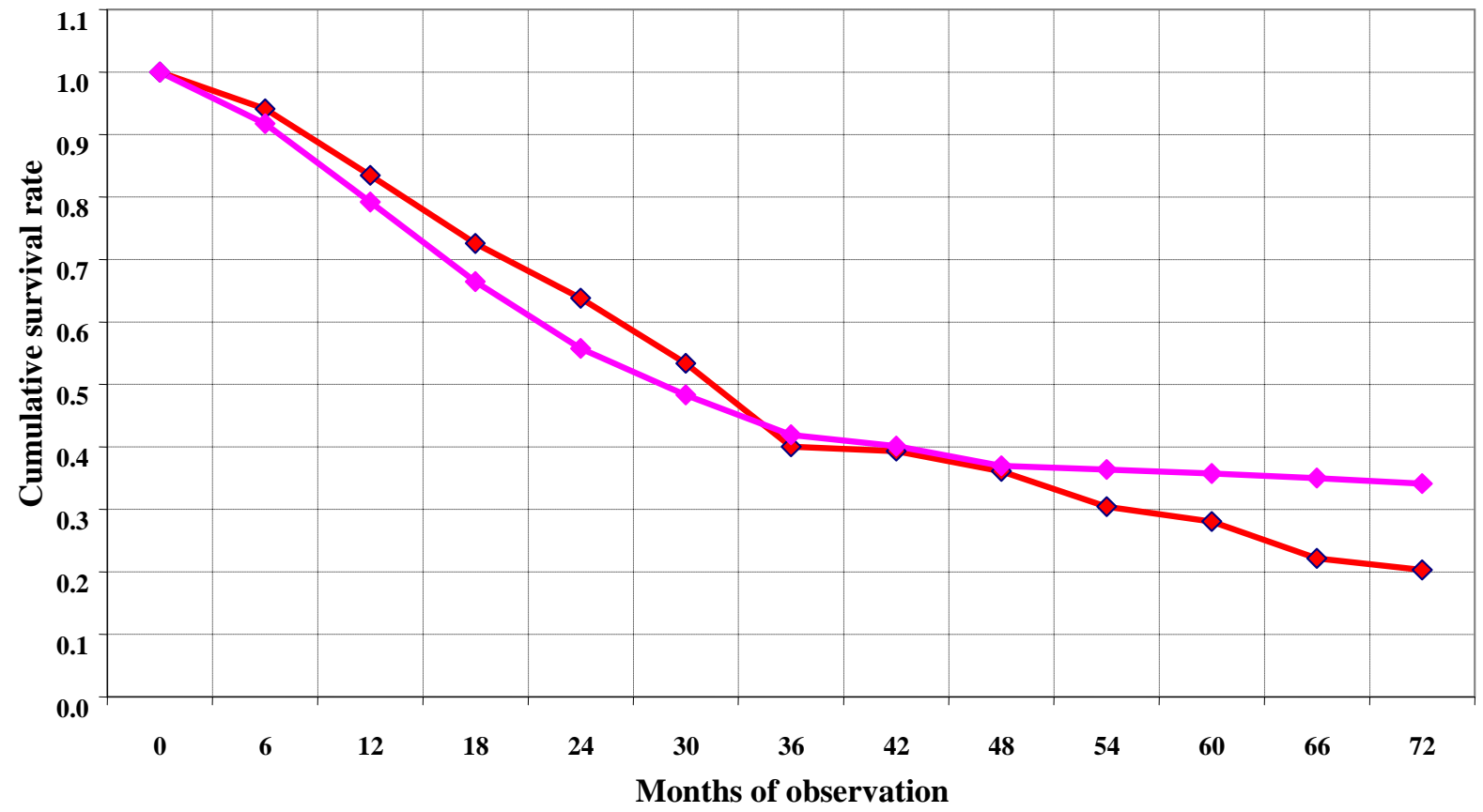

Negative PR

$\checkmark$ Positive PR

Fig. 8 Relapse-free survival of patients with serous OC with expression of progesterone receptor. Kaplan-Meier survival curves, log-rank criterion, $p=0.9534$.

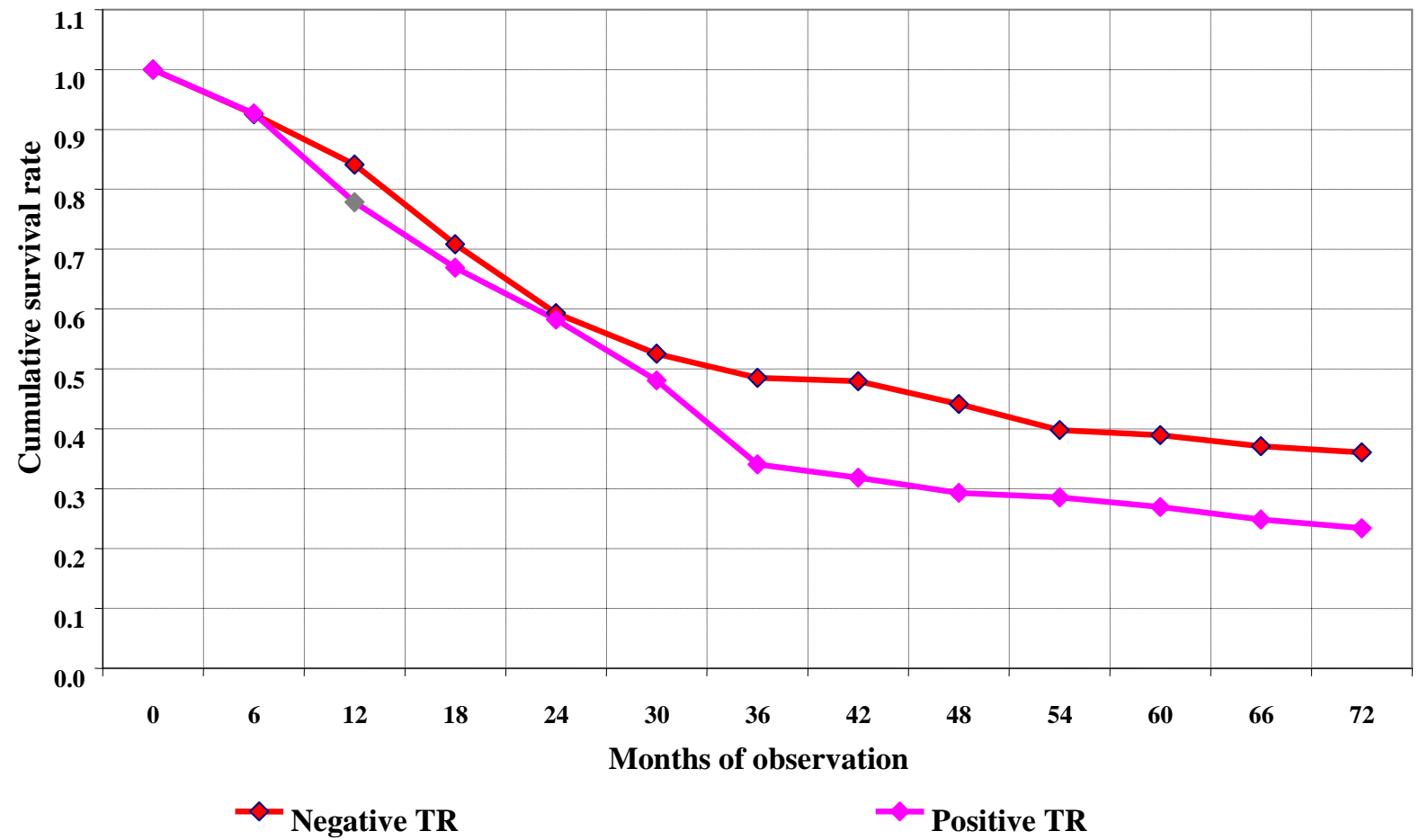

Fig. 9 Relapse-free survival of patients with serous OC with expression of testosterone receptor. Kaplan-Meier survival curves, log-rank criterion, $p<0.0500$. 
Table 7 Overall and relapse-free survival in patients with serous OC depending on expression of steroid hormone receptors.

\begin{tabular}{|c|c|c|c|c|c|c|}
\hline \multirow{3}{*}{$\begin{array}{l}\text { Steroid } \\
\text { hormone } \\
\text { receptors }\end{array}$} & \multicolumn{4}{|c|}{ Overall survival, \% } & \multicolumn{2}{|c|}{ Median of relapse-free survival, months } \\
\hline & \multicolumn{2}{|c|}{ Serous OC R + } & \multicolumn{2}{|c|}{ Serous OC R - } & \multirow{2}{*}{ Serous OC R + } & \multirow{2}{*}{ Serous OC R - } \\
\hline & 3-year & 5-year & 3-year & 5-year & & \\
\hline ER & $48.2 \pm 4.2$ & $39.3 \pm 4.4$ & $63.4 \pm 5.7$ & $39.3 \pm 6.4$ & $26.9 \pm 2.3$ & $34.8 \pm 2.7$ \\
\hline PR & $49.7 \pm 4.3$ & $39.3 \pm 4.4$ & $60.0 \pm 5.5$ & $39.3 \pm 6.1$ & $27.8 \pm 2.4$ & $32.0 \pm 2.5$ \\
\hline TR & $45.7 \pm 4.7$ & $31.4 \pm 4.8$ & $62.2 \pm 4.8$ & $47.9 \pm 5.3$ & $27.7 \pm 2.2$ & $35.7 \pm 2.8$ \\
\hline
\end{tabular}

Serous OC R + -receptor-positive serous OC; Serous OC R - -receptor-negative serous OC.

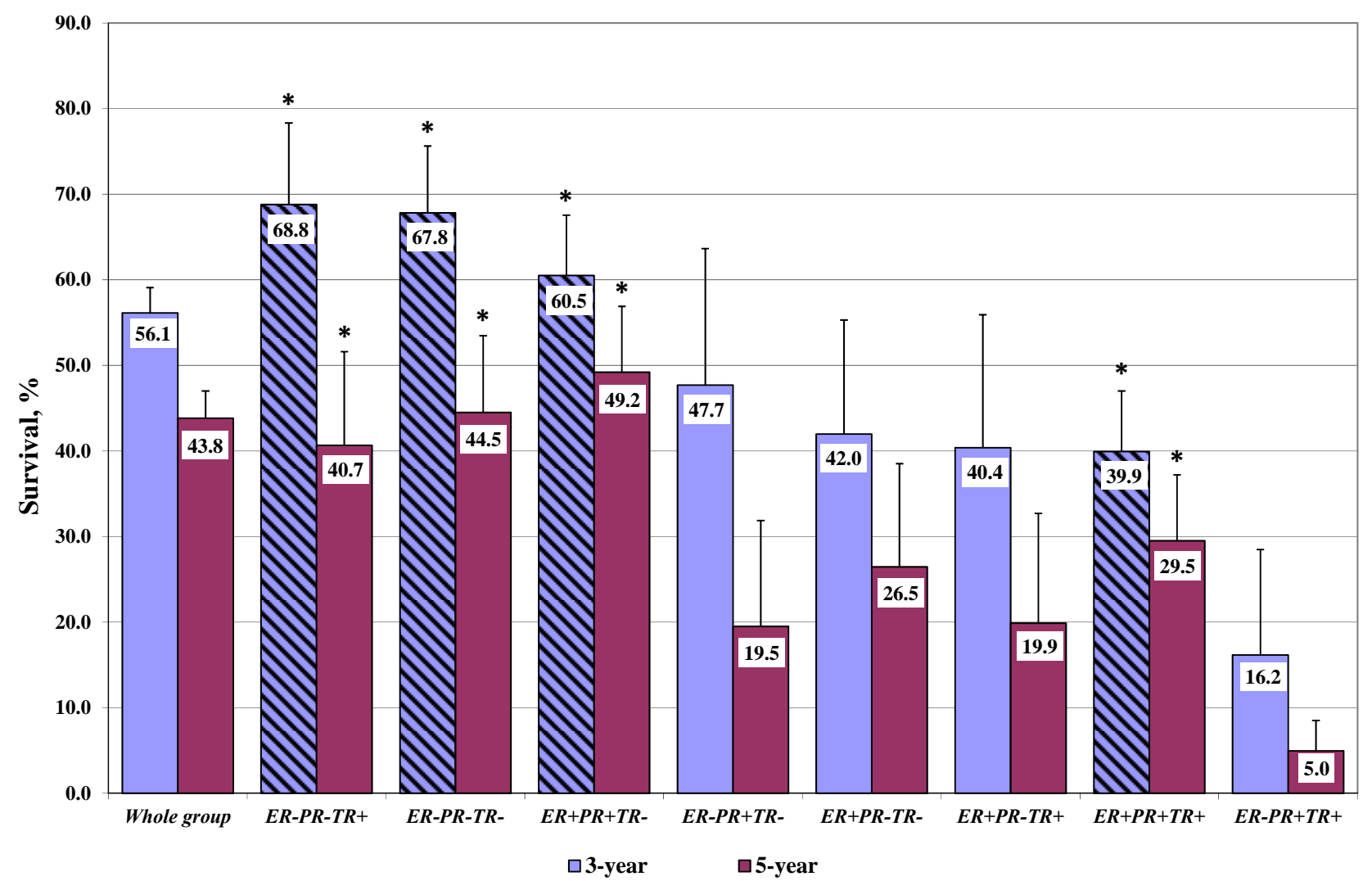

Fig. 10 Overall survival of patients with serous OC depending on tumor phenotype.

$* p<0.05$ if 3 -year and 5-year groups are compared.

receptors had higher 3-year and 5-year survival rates, than patients who had positive HRS of tumor: $67.8 / 44.5 \%$ and $39.9 / 29.5 \%$, respectively. In patients with tumor phenotype $\mathrm{ER}+\mathrm{PR}+\mathrm{TR}-$, rates of 3-year and 5-year survival have constituted 60.5/49.2\% compared to $39.9 / 29.5 \%$ - rates of overall survival of patients with positive HRS OC. However, in a group of patients with phenotype tumor which was positive only by TR, high survival rates have also been recorded $(60.5 / 49.5 \%)$ compared to the same in OC patients that was a positive tumor by all steroid hormone receptors $(39.9 / 29.5 \%)$. Thus, the lowest survival rates have been observed in patients who had positive HRS tumors.

When evaluating relapse-free survival of patients depending on serous OC phenotype (Fig. 11), significant intergroup differences only for tumors with phenotypes $\mathrm{ER}+\mathrm{PR}+\mathrm{TR}+$ and $\mathrm{ER}+\mathrm{PR}+\mathrm{TR}$ - have been obtained $(p<0.050)$. The highest rate of relapse-free survival has been determined in patients with $\mathrm{ER}+\mathrm{PR}+\mathrm{TR}-\mathrm{OC}$ phenotype (50.8/43.3\%) if compared with survival of the patients who had positive HRS (33.5/25.5\%), the absence of TR expression played the key role. 


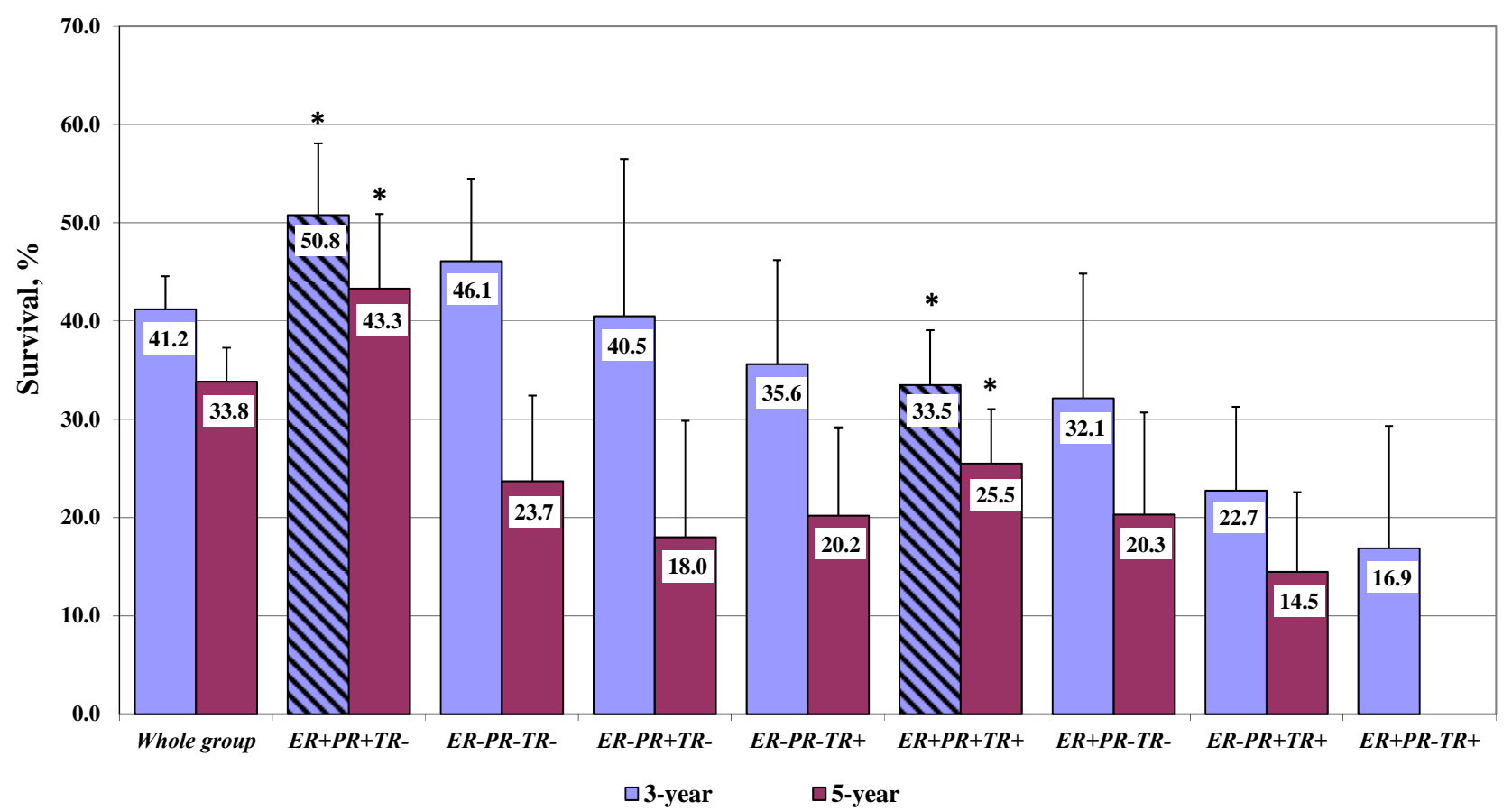

Fig. 11 Relapse-free survival of patients with serous OC depending on tumor phenotype.

$* p<0.05$ if 3-year and 5-year groups are compared.

Table 8 The rates of Cox-regression analysis of overall and relapse-free survival among the patients with serous OC depending on expression of the receptors to steroid hormones and hormonal receptor phenotype of tumor.

\begin{tabular}{|c|c|c|c|c|}
\hline \multirow{2}{*}{ Receptor status of serous OC } & \multirow{2}{*}{ HR } & \multicolumn{2}{|c|}{$95,0 \%$, CI } & \multirow{2}{*}{$p$} \\
\hline & & Lower & Higher & \\
\hline \multicolumn{5}{|c|}{ Overall survival } \\
\hline ER+ vs.ER- & 1.3 & 1.0 & 1.8 & 0.24 \\
\hline $\mathrm{PR}+$ vs.PR- & 1.2 & 0.4 & 1.5 & 0.25 \\
\hline TR+ vs. TR- & 1.3 & 1.1 & 1.9 & 0.01 \\
\hline $\mathrm{ER}+\mathrm{PR}+\mathrm{TR}+$ vs. ER-PR-TR- & 1.4 & 1.1 & 1.8 & 0.04 \\
\hline $\mathrm{ER}+\mathrm{PR}+\mathrm{TR}+$ vs. $\mathrm{ER}+\mathrm{PR}+\mathrm{TR}-$ & 1.4 & 1.1 & 1.8 & 0.04 \\
\hline ER-PR-TR+ vs. ER+PR+TR+ & 0.8 & 0.6 & 1.0 & 0.01 \\
\hline \multicolumn{5}{|c|}{ Relapse-free survival } \\
\hline ER+ vs.ER- & 1.2 & 0.8 & 1.5 & 0.23 \\
\hline PR+ vs.PR- & 1.2 & 0.5 & 1.6 & 0.15 \\
\hline TR+ vs. TR- & 1.3 & 0.9 & 1.7 & 0.04 \\
\hline $\mathrm{ER}+\mathrm{PR}+\mathrm{TR}+$ vs. ER-PR-TR- & 1.4 & 1.1 & 1.7 & 0.04 \\
\hline $\mathrm{ER}+\mathrm{PR}+\mathrm{TR}+$ vs. $\mathrm{ER}+\mathrm{PR}+\mathrm{TR}-$ & 1.1 & 0.5 & 1.8 & 0.20 \\
\hline
\end{tabular}

HR-hazard ratio-the ratio of risks of survival between the compared groups of patients; CI-confidence interval.

From evaluation of prognostic value of HRS in the patients with serous OC using the Cox-regression model (Table 8), it has been established that expression of TR in ovarian tumor increases the risk of disease recurrence (HR 1.3; 95.0\% CI 0.9-1.7) and is an unfavorable factor for the course of tumor process (HR 1.3; 95.0\% CI 1.1-1.7).
At multifactor analysis the risk of disease relapse is significantly higher (HR 1.4; $95.0 \%$ CI 1.1-1.7), whereas overall survival is lower in the patients with the receptor-positive phenotype of serous OC (HR 1.4; 95.0\% CI 1.1-1.8), than the same in the patients with negative HRS of the tumor (Table 8). In addition, significantly higher overall survival rates in patients 
with phenotype of serous OC ER-PR-TR+ and relapse-free survival in patients with phenotype of tumor $\mathrm{ER}+\mathrm{PR}+\mathrm{TR}-$ in comparison with survival rates in the patients with positive HRS of OC proves that expression of $\mathrm{TR}$ is an independent factor for unfavorable course of tumor process.

Represented results have demonstrated significant correlation between ER, PR, and TR in MOT that corresponds to data of some studies carried out before [37]. It is known that steroid hormones are able not only to regulate the level of own receptors, but also to modulate content of receptors of the other hormones. For instance, estradiol stimulates the formation of progesterone receptors, while progesterone decreases expression of estrogen receptors in reproductive organs. Moreover, estradiol is able to several times increase the level of ovarian androgenic receptors [38].

The highest frequency of receptor-positive tumors has been observed in patients with serous OC of postmenopausal period, which by own observations and data of literature is characterized by aggressive course and unfavorable prognosis of disease [2]. Such similarity by clinical course and tumor HRS is being detected also in patients with $\mathrm{BC}$ of postmenopausal period, but, at luminal A type of tumor in $\mathrm{BC}$ patients, the favorable prognosis of disease is being predicted $[39,40]$. Thus, the highest number of patients with serous OC with positive HRS has been observed in late postmenopausal period. It is the evidence of possible high sensitivity of tumor cells in patients of this age category to the impact of steroid hormones, most of which are formed due to extragonadal aromatization of androgens [6, 41, 42].

Univariate analysis has shown that overall and relapse-free survival rates are higher in patients with receptor-negative tumor by ER, PR and TR than with receptor-positive tumors, but significant differences have been registered only for TR.

Represented results have demonstrated low overall and relapse-free survival in patients with $\mathrm{OC}$ phenotype, which was receptor-positive for all steroid hormones. The highest rates of overall 5-year survival have been determined in patients with not only tumor phenotype ER-PR-TR-, but also with ER+PR+TR-. Relapse-free survival also was the highest in patients with serous OC phenotype ER+PR+TR- that indicates certain role of TR in clinical course of tumor process. Furthermore, with consideration of the results after a single-factor analysis of Cox-regression model, expression to TR must be regarded as an independent factor of unfavorable course of tumor process. Multifactor analysis of Cox-regression model proved that a positive hormonal receptor phenotype of serous $\mathrm{OC}$ is also the factor of unfavorable clinical course of tumor process.

\section{Discussion}

Obtained results, on one hand, correspond to the data of some studies which have demonstrated that strong expression of androgen receptors is being observed in case of invasive OC, and hyperandrogenia can be a risk factor of occurrence and progression of this disease [43]. On the other hand, results of our study contradict the data of the other studies which have determined that high expression of androgen receptors is a favorable prognostic factor in patients with serous OC [30, 44].

Today it is known that androgens interacting with receptors cause strong proliferative processes in ovarian tissue (hyperplasia of stroma, thecal tissue and cells of hilum of ovary) at endocrine syndromes of reproductive system in particular, polycystic ovary syndrome. Moreover, the level of androgens and expression of receptors at these syndromes are able to increase synchronously with a lapse of time [45]. Postmenopausal ovary is an androgenic ovary, stroma of which is the only source of gonadal hormone production in this age period. It has been proved that concentration of testosterone in ovarian vein is 15 times higher than the level of the latter in blood serum from peripheral vein [46]. As it has been stated above, 
peripheral synthesis of non-classic estrogens (phenol steroids) becomes more intensive in postmenopause. These estrogens possess weaker inhibiting effect on hypothalamus-hypophysis system than classic estrogens [6], and increase of gonadotropins production stipulates hyperplasia of ovarian thecal tissue that, in turn, can intensify synthesis of androgens [36, 45]. Non-classic phenol steroids and estrone are the main hormones in postmenopause. They are formed as the result of aromatization from androstenedione, secretion of which in menopause is mostly provided by adrenal glands and less-by ovaries [36, 45]. Phenol steroids are strong agonists of estradiol. Activity of these metabolites increases activity of the latter several times (in particular, 16- $\alpha$-hydroxyestrone 1 over 8 times), they form stable connections with specific receptors of cells of different tissues [47, 48].

It has been proved that transformation of androstenedione in estrone by percentage correlates with weight of women. Entering estrogens through aromatization of androgens is not limited only by fat tissue-almost all tissues of organism have this property. ER ( $\alpha$ and $\beta$ ) are found in brain and blood vessels, heart, bones, mammary glands, uterus, ovaries. ER- $\beta$ were detected only in lungs, kidneys, urinary bladder and intestine. Extragonadal estrogen- and androgen-formation serves as a source of additional hormonal stimulation [6, 45, 47, 48].

Among age endocrine-metabolic changes in women, which are manifested by obesity and insulin-resistance, compensatory hyperinsulinemia arises [7]. Insulin indirectly via receptors of insulin-like growth factor I the same as luteinizing hormone intensifies enzyme activity of biosynthesis of androgens in ovaries that causes certain increase of their functions [45]. It has been proved for today that androgen-regulating genes stimulate expression of epidermal growth factor, vascular endothelial growth factor and cyclin-dependent kinases 2 and 4. At the same time, they have repressing effect on the expression of TGF- $\beta$ and Bcl-2 [38].

In postmenopause along with a switching off the ovulatory function of ovaries a strong switching on the series of compensatory mechanisms takes place providing sufficient level and wide range of biologically active metabolites and at certain factors there are all necessary conditions for stimulation of target organs by them [6].

Thus, obtained results have demonstrated that endocrine factors and hormonal-receptor changes in ovaries are considerable elements of not only pathogenesis but also important prognostic criteria in postmenopausal patients with MOT. This represents an integral part of general mechanism of complicated molecular-genetic interactions.

\section{Conclusions}

Positive HRS has been determined in serous, mucinous $\mathrm{OC}$ and in SCST. High percentage of tumors with the expression of all steroid hormone receptors has been observed in them. However, the highest percentage of receptor-positive tumors has been detected in patients with mucinous $\mathrm{OC}$ and SCST that is confirmed by established correlation between morphological structure of tumors and expression of steroid hormone receptors.

Strong correlations of ER, PR expression in MOT with age period of patients as well as poor correlation with the stage of disease were established. Lack of impact of neoadjuvant chemotherapy on the expression of steroid hormone receptors was shown and no correlation with the differentiation grade of ovarian tumors of different genesis was determined.

In patients with serous $\mathrm{OC}$ of late menopausal period, the highest frequency of tumors with positive HRS has been recorded. Strong correlation between hormonal-receptor phenotype of serous OC and age period of patients was established $(r=0.5, p=0.002)$ which allows assuming that there is a high sensitivity of these tumors to both endogenous steroids and hormonal therapy. 
Strong correlation between ER, TR expression and relapse of serous OC was established. Univariate analysis has shown significantly low overall and relapse-free survival of the OC patients with tumor TR expression.

Multifactor analysis has established significantly low overall survival of patients with positive hormonal-receptor phenotype of serous OC (29.5\%) as compared to the one in patients with negative hormonal-receptor phenotype of tumor (44.5\%) $(p<$ 0.05).

Positive HRS in serous $\mathrm{OC}$ is the factor of unfavorable course of tumor process (HR 1.4; 95\%, CI 1.1-1.8).

HRS of MOT can be regarded as the additional criterion for solution of a question concerning application of hormonal therapy as a component of complex treatment for the patients and this demands further large multi-center studies in this direction.

\section{References}

[1] Heintz, A. P. M., Odicino, F., Maisonneuve, P., et al. 2006. "Carcinoma of the Ovary FIGO 6th Annual Report on the Results of Treatment in Gynecological Cancer." Int. J Gynaecol Obstet 95 (1): 161-9.

[2] Disaia, P. J., and Creasman, W. T. 2012. "Clinical Oncologic Gynecology.” M.: Rid Elsiver, 346.

[3] Vorobyova, L. I. 2012. "Practical Oncogynecology." Monograph: K, 228.

[4] Urmancheyeva, A. F., Kutusheva, G. F., and Ulrich, E. A. 2012. Ovarian Tumors (Clinics, Diagnostics and Treatment). St. Petersburg: N-L.

[5] Svintsitsky, V. S. 2004. "Ovarian Cancer: Dynamics of Certain Endocrinological Parameters under the Complex Therapy Effect." Clinical Endocrinology and Endocrine Surgery 3 (8): 25-30.

[6] Vorobyova, L. I., Svintsitsky, V. S., and Tkalia, I. G. 2013. "Hormonal Carcinogenesis and Substantiation of Application of Hormonal Therapy in Treatment of Ovarian Cancer." Clin Oncol 1 (9): 56-64.

[7] Landen, J. C. N., Birrer, M. J., and Sood, A. K. 2008. "Early Stages of the Pathogenesis of Ovarian Cancer." J Clin Oncol 26 (6): 149-60.

[8] Vorobyova, L. I., and Tkalia, I. G. 2013. "Clinical Significance of Concomitant Hyperplastic Processes of Endometrium in Patients with Malignant Ovarian Tumors." Oncology 4 (58): 286-93.
[9] Blanco, L. Z., Jr, Kuhn, E., Morrison, J. C., et al. 2017. "Steroid Hormone Synthesis by the Ovarian Stroma Surrounding Epithelial Ovarian Tumors: A Potential Mechanism in Ovarian Tumorigenesis." Mod Pathol 30 (4): 563-76.

[10] Bondar, G. V., Lisovska, N. Y., Kajriak, O. V., et al. 2009. "Chemohormonotherapy in the Combined Treatment of Advanced Ovarian Cancers." Problems of Modern Medical Science and Education 2: 33-35.

[11] Gorbunova, V. A. 2011. "Diagnostics and Treatment of Ovarian Cancer.” M: MIA, 248.

[12] Perevodchikova, N. I. 2011. "Guidelines for Chemotherapy of Tumors." Practical Medicine, 512.

[13] Bookman, M. A., Brady, M. F., McGuire, W. P., et al. 2009. "Evaluation of New Platinum-Based Treatment Regimens in Advanced-Stage Ovarian Cancer: A Phase III Trial of the Gynecologic Cancer Intergroup." J Clin Oncol 27: 1419-25.

[14] Bristow, R. E., Puri, I., and Chi, D. S. 2009. "Cytoreductive Surgery for Recurrent Ovarian Cancer: A Meta-Analysis." Gynecol Oncol 112: 265-74.

[15] Maksimov, S. Y., and Huseynov, K. D. 2010. "Targeted Therapy in Ovarian Cancer." Pract Oncol 11 (3): 54-64.

[16] Svintsitsky, V. S., and Vorobyova, L. I. 2010. "Malignant Ovarian Tumors: Optimization of Complex Treatment." Oncology 12 (1): 52-6.

[17] Cancer in Ukraine 2015-2016. "Incidence, mortality, activities of oncological service" Bulletin of National Cancer Registry of Ukraine 18: 48-49.

[18] Urmancheyeva, A. F., Tyulyandin, S. A., and Moiseyenko, V. M. 2008. Practical Oncogynecology: Selected Lectures. St. Petersburg: Center TOMM.

[19] King, E. R., and Wong, K. K. 2011. "Steroid Hormones and Ovarian Cancer." In Steroids-Clinical Aspect, edited by Hassan, A. Accessed. http://www.intechopen.com/books/steroids-clinical-aspec $\mathrm{t} /$ steroid-hormones-and-ovarian-cancer.

[20] Schepotin, I. B., Zotov, A. S., Anikusko, N. F., et al. 2011. "Neoadjuvant Hormonal Therapy of Locally Disseminated Breast Cancer." Clin Oncol 3 (3): 36-9.

[21] Burges, A., Brüning, A., Dannenmann, C., et al. 2010. "Prognostic Signi Wcance of Estrogen Receptor Alpha and Beta Expression in Human Serous Carcinomas of the Ovary." Arch Gynecol Obstet 281: 511-7.

[22] Aust, S., Horak, P., Pils, D., et al. 2013. "The Prognostic Value of Estrogen Receptor Beta and Proline-, Glutamic Acid- and Leucine-rich Protein 1 (PELP1) Expression in Ovarian Cancer." BMC Cancer 13: 115.

[23] Halon, A., Materna, V., Drag-Zalesinska, M., et al. 2011. "Estrogen Receptor Alpha Expression in Ovarian Cancer Predicts Longer Overall Survival." Pathol Oncol Res 17 (3): 511-8. 
[24] Alonso, L., Gallego, E., Jesús González, F., et al. 2009. "Gonadotropin and Steroid Receptors as Prognostic Factors in Advanced Ovarian Cancer: Aretrospective Study." Clin Transl Oncol 11: 748-52.

[25] Ayadia, L., Chaabounia, S., Khabira, A., et al. 2010. "Correlation between Immunohistochemical Biomarkers Expression and Prognosis of Ovarian Carcinomas in Tunisian Patients." World J Oncol 1 (3): 118-28.

[26] Chakraborty, A., Chatterjee, S., and Roy, P. 2010. "Progesterone Receptor Agonists and Antagonists as Anticancer Agents." Mini Rev Med Chem 10 (6): 506-17.

[27] García-Velasco, A., Mendiola, C., Sánchez-Muñoz, A., et al. 2008. "Prognostic Value of Hormonal Receptors, P53, Ki67 and HER2/Neuexpression in Epithelial Ovarian Carcinoma." Clin Transl Oncol 10: 367-71.

[28] Nourieh, S., Zohreh, Y., Shohreh, S., et al. 2009. "Prognostic Values of Estrogen and Progesterone Expression Receptors in Ovarian Papillary Serous Carcinoma." Ir J Pathology 4 (1): 9-12.

[29] Valladares, M., Plaza-Parrochia, F., Lépez, M., et al. "Effect of Estradiol on the Expression of Angiogenic Factors in Epithelial Ovarian Cancer." Histol Histopathol 24: 11874. doi: 10.14670/HH-11-874.

[30] Jönsson, J. M., Skovbjerg Arildsen, N., Malander, S., et al. 2015. "Sex Steroid Hormone Receptor Expression Affects Ovarian Cancer Survival." Transl Oncol 8 (5): 424-33.

[31] van Kruchten, M., van der Marel, P., de Munck, L., et al. 2015. "Hormone Receptors as a Marker of Poor Survival in Epithelial Ovarian Cancer." Gynecol Oncol 138 (3): 634-9.

[32] Dudnichenko, A. S., Yakimova, T. P., and Kartashov, S. M. 2001. "Receptor Status of Ovary Cells Depending on the Morphological Features and Chemotherapeutic Effects." Oncology 3 (4): 271-4.

[33] Hsiao, T. H., Chiu, Y. C., Hsu, P. Y., et al. 2016. "Differential Network Analysis Reveals the Genome-Wide Landscape of Estrogen Receptor Modulation in Hormonal Cancers." Sci Rep 14 (6): 23035. doi: 10.1038/srep23035.

[34] Puzin, S. N., Payanidi, Y. G., Ogay, D. S., et al. 2012. "Medical Expertise in Oncogynecology." Oncogynecology 2: 60-7.

[35] Lenhard, M., Tereza, L., Heublein, S., et al. 2012.
"Steroid Hormone Receptor Expression in Ovarian Cancer: Progesterone Receptor B as Prognostic Marker for Patient Survival." BMC Cancer 12: 553.

[36] Manukhin, I. B., Tumilovich, L. G., and Gevorkyan, M. A. 2003. "Clinical Lectures on Gynecological Endocrinology." M: MIA, 247.

[37] Bokhman, Y. V. 2002. Guidelines in Oncogynecology. St. Petersburg: Foliant.

[38] Korman, D. B. 2010. "Endocrine Therapy of Malignant Tumors." M: Practical Medicine, 400.

[39] Schepotin, I. B., Zotov, A. S., Lyubota, R. V., et al. "Molecular Subtypes of Breast Cancer Determined on the Basis of Immunohistochemical Markers: Clinical and Biological Features and Prognosis of Course." Clin Oncol 8 (4): 1-4.

[40] Shafrir, A. L., Rice, M. S., Gupta, M., et al. 2016. “The Association between Reproductive and Hormonal Factors and Ovarian Cancer by Estrogen-A and Progesterone Receptor Status." Gynecol Oncol 143 (3): 628-35.

[41] Voutsadakis, I. A. 2016. "Hormone Receptors in Serous Ovarian Carcinoma: Prognosis, Pathogenesis, and Treatment Considerations." Clin Med Insights Oncol 29 (10): 17-25.

[42] Ren, Y. A., Mullany, L. K., Liu, Z., et al. 2016. "Mutant p53 Promotes Epithelial Ovarian Cancer by Regulating Tumor Differentiation, Metastasis, and Responsiveness to Steroid Hormones.” Cancer Res 76 (8): 2206-18.

[43] Wang, P. H., and Chang, C. 2004. "Androgens and Ovarian Cancers.” Eur J Gynaec Oncol 25 (2): 157-63.

[44] Nodin, B., Zendehrokh, N., Brändstedt, J., et al. 2010. "Increased Androgen Receptor Expression in Serous Carcinoma of the Ovary Is Associated with An Improved Survival." J Ovarian Res 3: 14.

[45] Vikhlyayeva, E. M. 2002. "Guide to the Endocrine Gynecology.” M: MIA, 768.

[46] Risch, H. A. 1998. "Hormonal Etiology of Epithelial Ovarian Cancer, with a Hypothesis Concerning the Role of Androgens and Progesterone (Review)." J Natl Cancer Inst 90: 1774-86.

[47] Dilman, V. M. 1987. "Four Models of Medicine." L: Medicine, 288.

[48] Berstein, L. M. 1998. Extragonadal Production of Estrogens. St. Petersburg: Nauka, 13-19. 